\title{
Efficient loading of dendritic cells following cryo and radiofrequency ablation in combination with immune modulation induces anti-tumour immunity
}

\author{
MHMGM den Brok ${ }^{*, 1,2}$, RPM Sutmuller', S Nierkens', EJ Bennink', C Frielink ${ }^{3}$, LWJ Toonen', OC Boerman², \\ CG Figdor', TJM Ruers ${ }^{2}$ and GJ Adema*,1
}

'Department of Tumor Immunology, Nijmegen Centre for Molecular Life Sciences, Radboud University Nijmegen Medical Centre, PO Box 9101 , Nijmegen 6500 HB, The Netherlands; '2Department of Surgery, Radboud University Nijmegen Medical Centre, PO Box 9101 , Nijmegen 6500 HB, The Netherlands; ${ }^{3}$ Department of Nuclear Medicine, Radboud University Nijmegen Medical Centre, PO Box 9I0I, Nijmegen 6500 HB, The Netherlands

Dendritic cells (DC) are professional antigen-presenting cells that play a pivotal role in the induction of immunity. Ex vivo-generated, tumour antigen-loaded mature DC are currently exploited as cancer vaccines in clinical studies. However, antigen loading and maturation of DC directly in vivo would greatly facilitate the application of DC-based vaccines. We formerly showed in murine models that radiofrequency-mediated tumour destruction can provide an antigen source for the in vivo induction of anti-tumour immunity, and we explored the role of DC herein. In this paper we evaluate radiofrequency and cryo ablation for their ability to provide an antigen source for DC and compare this with an ex vivo-loaded DC vaccine. The data obtained with model antigens demonstrate that upon tumour destruction by radiofrequency ablation, up to 7\% of the total draining lymph node (LN) DC contained antigen, whereas only few DC from the conventional vaccine reached the LN. Interestingly, following cryo ablation the amount of antigen-loaded DC is almost doubled. Analysis of surface markers revealed that both destruction methods were able to induce DC maturation. Finally, we show that in situ tumour ablation can be efficiently combined with immune modulation by anti-CTLA-4 antibodies or regulatory Tcell depletion. These combination treatments protected mice from the outgrowth of tumour challenges, and led to in vivo enhancement of tumour-specific T-cell numbers, which produced more IFN- $\gamma$ upon activation. Therefore, in situ tumour destruction in combination with immune modulation creates a unique, 'in situ DC-vaccine' that is readily applicable in the clinic without prior knowledge of tumour antigens.

British Journal of Cancer (2006) 95, 896-905. doi: I0.1038/sj.bjc.660334 I www.bjcancer.com

Published online 5 September 2006

(c) 2006 Cancer Research UK

Keywords: vaccination; dendritic cell; CTLA-4; regulatory T cell; radiofrequency ablation; cryo ablation

Dendritic cells (DC) are crucial antigen-presenting cells (APC) for the initiation of primary $\mathrm{T}$-cell responses (Adams et al, 2005; Banchereau and Palucka, 2005). Immature DC are well equipped to take up and process antigen from their surroundings, but they lack sufficient co-stimulatory signals required for productive T-cell activation. In a stimulatory environment, like in an infection, immature DC undergo activation, maturation and acquire the capacity to cross-present exogenous antigens in MHC class I. Particularly, the Toll-like receptor (TLR) family of proteins initiates the DC maturation process upon recognition of conserved pathogen-associated molecular patterns (PAMPs), like LPS or unmethylated $\mathrm{CpG}$ oligodeoxynucleotides. Upon maturation, costimulatory molecule and MHC-peptide complex expression increases and cytokines like IL-12 skew the functional outcome of

*Correspondence: Dr GJ Adema; E-mail: g.adema@ncmls.ru.nl; MHMGM den Brok, E-mail: m.denbrok@ncmls.ru.nl

Revised 19 July 2006; accepted 28 July 2006; published online 5 September 2006 the response (Trinchieri, 2003). Dendritic cells that did not perceive an activating environment while taking up antigen do not mature and induce tolerance rather than immunity. The importance of immune activation for the induction of anti-tumour immunity has been well established. Next to direct activation of DC by PAMPs, a broad range of indirect strategies has been explored to accomplish activation of the immune system. Expression of, for instance, costimulatory molecules on tumours can induce T-cell-mediated rejection of a variety of tumours (Townsend and Allison, 1993). In addition to these stimulatory pathways, also blockade of inhibitory receptors, for example, CTLA-4, has been applied successfully to induce tumour rejection (Chen et al, 1992; Egen et al, 2002). In this respect, also in vivo depletion of regulatory $\mathrm{T}$ cells, that are able to suppress conventional T-cell expansion, has been shown to evoke anti-tumour immunity (Sutmuller et al, 2001; Sakaguchi, 2005).

As murine models demonstrate that especially DC are effective in inducing effective immune responses, ex vivo generated DC are currently applied to stimulate anti-tumour immunity in clinical trials (Banchereau et al, 2000; Coulie and van der Bruggen, 2003; De Vries et al, 2003; Schuler et al, 2003; Figdor et al, 2004). 
Although tumour-specific responses have been obtained with tumour antigen-loaded DC-based vaccines, many questions remain unanswered (Figdor et al, 2004; Steinman and Mellman, 2004). Especially the migration of ex vivo generated DC-based vaccines from i.d./subcutaneously (s.c.) injected depots to the draining lymph nodes (LN) has been shown to be inefficient in both mouse models and patients (Eggert et al, 1999; Josien et al, 2000; De Vries et al, 2003). Moreover, ex vivo generation and loading of $\mathrm{DC}$ is time consuming and costly. In vivo loading and maturation of DC would therefore improve the applicability of DC vaccination to a great extend.

Recent studies using antigens coupled to antibodies directed against the mouse DC antigen DEC-205 (Steinman and Pope, 2002) or attraction of DC to the tumour via retrovirus-mediated expression of the DC-attracting chemokine CCL20 (Furumoto et $a l, 2004)$ illustrate the possibility to directly load tumour antigens onto DC in vivo. We previously showed in a murine model that the tumour debris left in the body after in situ tumour destruction by radiofrequency ablation is an in vivo tumour antigen source for the immune system. Excision of the ablated tumour largely prevented the induction of anti-tumour immunity (unpublished observation). Adoptive transfer experiments demonstrated that the immunity induced is T-cell-dependent. Dendritic cells appeared to play an important role in the initiation of this immune response. Tumour ablative treatments, like cryo or radiofrequency ablation, are successfully used in clinical settings to destruct different types of tumours (Zagoria et al, 2001; Ruers and Bleichrodt, 2002; Curley, 2003; Garcea et al, 2003; Raj et al, 2003). Nevertheless, cancer patients treated with ablative regimens mostly develop systemic recurrences as a consequence of the outgrowth of distant micro-metastases, implying that in general no protective immune response is induced. This observation is consistent with our findings that efficient induction of immunity following ablation requires that additional immune activation stimuli are given simultaneously (den Brok et al, 2004).

We now show that in situ tumour destruction by either cryo or radiofrequency ablation can be employed to efficiently provide antigens to DC in vivo. A side-by-side comparison of the two ablative techniques demonstrates that following cryo ablation of established B16 tumours $(5-7 \mathrm{~mm})$ up to $13 \%$ of DC in the draining LN acquire tracer proteins as a marker of tumour-derived antigen. Radiofrequency ablation results in $7 \%$ of LN DC being loaded with the marker antigen. With both ablation methods far more marker antigen-containing DC are detected in the draining $\mathrm{LN}$ than with a conventional in vitro generated $\mathrm{DC}$ vaccine. We further show that both destruction methods in itself were able to enhance DC maturation in vivo to an equal extend, comparable to ex vivo maturation of DC. Finally, we report that both tumour ablation techniques can be efficiently combined with immunomodulatory approaches, like blockade of CTLA-4 signalling or regulatory $\mathrm{T}$-cell depletion, to induce functional $\mathrm{CD} 8+\mathrm{T}$ cells creating systemic anti-tumour-immunity.

Therefore, in situ tumour destruction by cryo or radiofrequency ablation combined with immuno-modulatory approaches constitutes a powerful 'in situ DC-vaccine' for which no prior knowledge of tumour antigens is needed.

\section{MATERIALS AND METHODS}

\section{Animals}

Nine- to 11-week-old female C57BL/6n mice were purchased from Charles River Wiga (Sulzfeld, Germany). Animals were held under specified pathogen-free conditions in the Central Animal Laboratory (Nijmegen, The Netherlands). All experiments were performed according to the guidelines for animal care of the Nijmegen Animal Experiments Committee.

\section{Tumours}

Mice were injected s.c. at the right femur with $500 \times 10^{3}$ cells of the OVA-transfected murine melanoma cell line B16F10 (B16-OVA, clone MO5), which was kindly provided by Dr Kenneth Rock (Falo et al, 1995), or wt B16F10. Cells were cultured and injected as described before (den Brok et al, 2004). Tumour volumes were scored every 3 days with the formula $\left(\mathrm{AxB}^{2}\right) \times 0.4$, in which $\mathrm{A}$ is the largest and $\mathrm{B}$ is the shortest dimension. Tumours were selected for ablation when their diameter measured between 5 and $7 \mathrm{~mm}$ $(\mathrm{d} 9-10)$ and only if the tumour was relatively round $(>98 \%$ of mice).

\section{Radiofrequency ablation}

Animals were anaesthetised by isoflurane inhalation and properly shaved at the tumour area and on the contra-lateral flank. After placement and proper attachment of the contra lateral side onto an electricity-conducting pad (grounding pad), the tumour area was disinfected with alcohol. A radiofrequency ablation needle with active tip of $8 \mathrm{~mm}$ (SMK-15, Cotop, Amsterdam, The Netherlands) was inserted s.c. and placed in the middle of the tumour. After placement of the radiofrequency ablation needle, impedance could be evaluated on the radiofrequency lesion generator system (Model RFG-3B, Radionics, Burlington, MA, USA). Next, treatment was started by delivering radiofrequency energy. During a treatment cycle of $\pm 80 \mathrm{~s}$, temperature could be monitored by means of a thermistor and thermocouple in the tip of the probe. Treatment was considered successful if a tip temperature of $75-80^{\circ} \mathrm{C}$ could be reached.

\section{Cryo ablation}

Animals were properly shaven and anaesthetised by isoflurane inhalation. The tumour area was disinfected with alcohol and subsequently wetted with distilled water. The tip of the liquid nitrogen cryo ablation system (CS76, Frigitronics, Shelton, CT, USA) was placed onto the tumour and after proper freeze attachment, treatment was started. During two treatment cycles of $\pm 70 \mathrm{~s}$ the tumour and a small strip around it were frozen to less than $-100^{\circ} \mathrm{C}$. Treatment was considered successful when the whole tumour appeared frozen macroscopically.

\section{Re-challenge model}

Forty days after ablation of B16-OVA tumours, mice were challenged by s.c. injection at the contra-lateral femur of either $15 \times 10^{3}$ B16-OVA cells or $15 \times 10^{3}$ EL4 cells (numbers defined by titration). Injections were performed in $100 \mu \mathrm{l}$ PBS. Mice were killed when tumours reached a volume of $\pm 850 \mathrm{~mm}^{3}$.

\section{${ }^{111}$ Indium conjugation and antigen monitoring}

KLH (Calbiochem, Darmstadt, Germany) $\left(10 \mathrm{mg} \mathrm{ml}^{-1}\right)$ was conjugated with $240 \mu \mathrm{g}$ cDTPA (Sigma-Aldrich, Zwijndrecht, The Netherlands) in $0.1 \mathrm{M} \mathrm{NaHCO}_{3}, \mathrm{pH} 8.2$, during $30 \mathrm{~min}$. Unconjugated DTPA was removed by dialysis against $0.1 \mathrm{M}$ Na-citrate buffer, $\mathrm{pH}$ 5.0. The KLH-DTPA conjugate $(1.5 \mathrm{mg})$ was incubated with $1.5 \mathrm{mCi}^{111} \mathrm{InCl}_{3}$ (Mallinckrodt, Petten, The Netherlands) in $1.2 \mathrm{ml} 0.1 \mathrm{M} \mathrm{Na}$-citrate buffer, pH 5.0, during $30 \mathrm{~min}$. Similar procedures were followed for the ${ }^{111}$ In-OVA conjugate. Radiochemical purity of each preparation $(>95 \%)$ was determined by instant thin-layer chromatography (ITLC, Gelman Sciences Inc., MI, USA). For antigen monitoring experiments, mice received intratumoural (i.t.) injections of OVA/KLH labelled with ${ }^{111} \mathrm{In}$ $\left({ }^{111} \mathrm{In}-\mathrm{OVA},{ }^{111} \mathrm{In}-\mathrm{KLH}\right)$ or ovalbumin conjugated to Alexa-488 (OVA-Alexa-488) (Molecular Probes, Leiden, The Netherlands). Conjugates $(20 \mu \mathrm{g}(=20 \mu \mathrm{Ci}))$ were injected directly before 
ablation in $20 \mu \mathrm{l}$ PBS. At various time points after injection of the protein conjugates, mice were anesthetised and scintigraphic images were acquired using a $\gamma$-camera with ${ }^{111}$ In collimator (Siemens Orbiter, Siemens Inc. Hoffmann Estate, IL, USA) as described previously (Eggert et al, 1999). For biodistribution studies mice were killed at different time points after injection of the protein conjugates. Liver, kidney, spleen, draining LN (r. sup. ing.) and non-draining LN (l. sup. ing.) were collected, weighed and counted in a $\gamma$-counter (1480 Wizard, Wallac Oy, Finland). Injection standards were taken for physical decay correction. In DC sorting experiments, mice received ${ }^{111}$ In-KLH i.t., and at different time points post-ablation CD11c + DC were sorted as described below and counted in the $\gamma$-counter.

\section{Magnetic bead cell sorting and flow cytometric analysis}

For antigen uptake experiments ( ${ }^{111} \mathrm{In}-\mathrm{KLH}$ and OVA-Alexa), draining LN's from five to eight mice were pooled and after crushing, dissociation in DNAse/collagenase/EDTA, and passage through nylon mesh (Vremec et al, 2000), cells were counted and sorted by standard MACS isolation with a MACS Midi column (Miltenyi Biotec). Positive selection of DC was done using CD11c beads (clone N418, Miltenyi Biotec, B.Gladbach, Germany), whereas negative selection/enrichment was done on the CD90 Tcell marker (Thy1.2, 30.H12, Miltenyi Biotec). Sorts were verified by CD3e or CD11c (HL3) staining (not shown). Subsequently, cells were stained and analysed on a FACS-Calibur ${ }^{\mathrm{TM}}$ system (BD) with the CELLQuest software. Stainings were performed using the following mAbs: CD11c-APC (HL3), CD8b-FITC (53-5.8), CD3ePE (17-A2), biotinylated CD80 (1G10) and streptavidin-PE. All antibodies were purchased from BD Pharmingen (Alphen a/d Rijn, The Netherlands).

\section{Dendritic cell culture from green fluorescent protein transgenic mice}

GFP-expressing DC were cultured and injected as described elsewhere (Eggert et al, 2003). Briefly, bone marrow was collected from GFP-transgenic mice and cultured for 7 days in the presence of GM-CSF and IL-4. At day 7, $1 \mu \mathrm{g} \mathrm{ml}^{-1}$ LPS was added for $24 \mathrm{~h}$ maturation. Next, the non-adherent fraction was harvested, washed and loaded for $1 \mathrm{~h}$ with the $\mathrm{K}^{\mathrm{b}}$-peptide of OVA (SIINFEKL). Cells $\left(1 \times 10^{6}\right)$ were injected s.c. or peri-tumourally (p.t.) at the femur. Isolation of LN cells and sorting was identical as described above.

\section{Antibody treatments}

In the anti-CTLA- 4 treatment mice received anti-CTLA- 4 antibody (clone 9H10 (Egen et al, 2002)) at days 0, 3 and 6 after ablation. Treg depletion was performed by injection of anti-CD25 antibodies (clone PC61, (Onizuka et al, 1999)) 4 days before ablation. Injections $(200 \mu \mathrm{g})$ were done intraperitoneal in PBS. In all cases depletion was successful as verified by FACS (not shown).

\section{Tetramer staining and interferon- $\gamma$ measurement}

A T-cell culture was obtained from spleen and draining LN's of mice 10 days after ablation of a B16-OVA tumour or from naïve control mice. Stimulation of these cells $\left(100 \times 10^{3}\right)$ was performed by addition of irradiated, IFN- $\gamma$-treated, B16-OVA cells $\left(50 \times 10^{3}\right)$ in IL-2 $\left(10 \mathrm{CU} \mathrm{ml}^{-1}\right)$ supplemented culture medium. At days 5 and 10 , cells were collected and cleaned in a density gradient. At day 10 of culture, cells were stained for $15 \mathrm{~min}$ at RT by OVA-tetramers $\left(\mathrm{H}-2 \mathrm{~K}^{\mathrm{b}}\right)$ conjugated to APC (Pelimers, Sanquin, Amsterdam, The Netherlands), counterstained with CD8b, and analysed by FACS. Same bulk cultures were used to collect supernatant $24 \mathrm{~h}$ after stimulation with irradiated B16-OVA cells. Capture and biotiny- lated detection antibodies directed to mouse IFN $-\gamma$ were purchased from BD Pharmingen and, using standard ELISA procedures, IFN$\gamma$ concentration was measured in $50 \mu$ l of supernatant.

\section{Statistical analysis}

Data were analysed for statistical significance by Student's $t$ test, except for the Kaplan-Meier survival curves for which a log rank test was used.

\section{RESULTS}

\section{Immune responses following radiofrequency or cryo ablation}

We previously demonstrated that radiofrequency ablation of established $(5-7 \mathrm{~mm})$ murine tumours resulted in weak, but tumour-specific anti-tumour reactivity. However, the mechanism by which immunity is induced and the role of DC herein remain largely unknown. Therefore, we explored the fate of tumour debris generated by two distinct tumour ablation approaches and the role of DC in the subsequent induction of immune responses. To first compare the induction of immunity after both techniques, B16OVA tumour-bearing mice were treated with either radiofrequency or cryo ablation and then re-challenged with either B16-OVA cells or non-related EL4 thymoma cells. A detailed time schedule is given below Figure 1. Re-challenges were given 40 days after ablation to exclude direct effects of the ablations on the tumour rechallenge. As shown in Figure 1, radiofrequency ablation of B16OVA resulted in a clear delay in the outgrowth of B16-OVA tumour cells as compared to naïve controls and a low level of protection ( $20 \%$ of the mice, lower right panel). Interestingly, when mice received cryo ablation, slightly more mice were protected $(50 \%$ of the mice, upper right panel). In contrast, no delay in outgrowth of the non-related EL4 mouse thymoma was observed (left panels). These data imply that a weak, but tumourspecific immune response had developed after both in situ tumour destruction techniques. In all experiments, cryo ablation was slightly more effective than radiofrequency ablation.

\section{Efficient in vivo antigen acquisition by lymph node CD11c $(+)$ dendritic cell following ablation}

To determine the fate of tumour antigens and the involvement of DC in the observed anti-tumour immune responses, we studied in vivo antigen acquisition by $\mathrm{DC}$ following radiofrequency and cryo ablation. Mice carrying established B16-OVA tumours (5-7 mm) received an i.t. injection of ${ }^{111}$ Indium-labelled KLH or ${ }^{111}$ In-OVA tracer proteins before ablation to monitor the fate of tumourdebris. $\gamma$-Camera imaging demonstrated that ${ }^{111}$ In proteins remained in the tumour for at least $72 \mathrm{~h}$, whereas unbound ${ }^{111} \mathrm{InCl}_{3}$ rapidly distributes throughout the mice (Figure $2 \mathrm{~A}$ ). Even following both ablation procedures ${ }^{111} \mathrm{In}-\mathrm{KLH}$ and OVA largely remained at the treated site whereas only minor local spreading was observed. In case ${ }^{111}$ In-OVA was used slightly more spreading to the liver was seen compared to $\mathrm{KLH}$, which is likely dependent on the molecular characteristics of OVA. Biodistribution analysis revealed an ablation-dependent accumulation of radioactivity in the draining LN's when ${ }^{111}$ In-KLH (Figure $2 \mathrm{~B}-\mathrm{D}$ ) or ${ }^{111} \mathrm{In}-\mathrm{OVA}$ (not shown) was used. Although significantly different from untreated tumour-bearing mice, radiofrequency-ablated mice showed significantly less accumulation of radioactivity compared to cryo ablation (Figure 2B). Little or no activity was found in the non-draining LN's, liver, kidneys or spleen with or without ablation (Figure 2C), whereas the organs contained high concentrations of radioactivity following injection of unbound ${ }^{111} \mathrm{InCl}_{3}$ (not shown). 

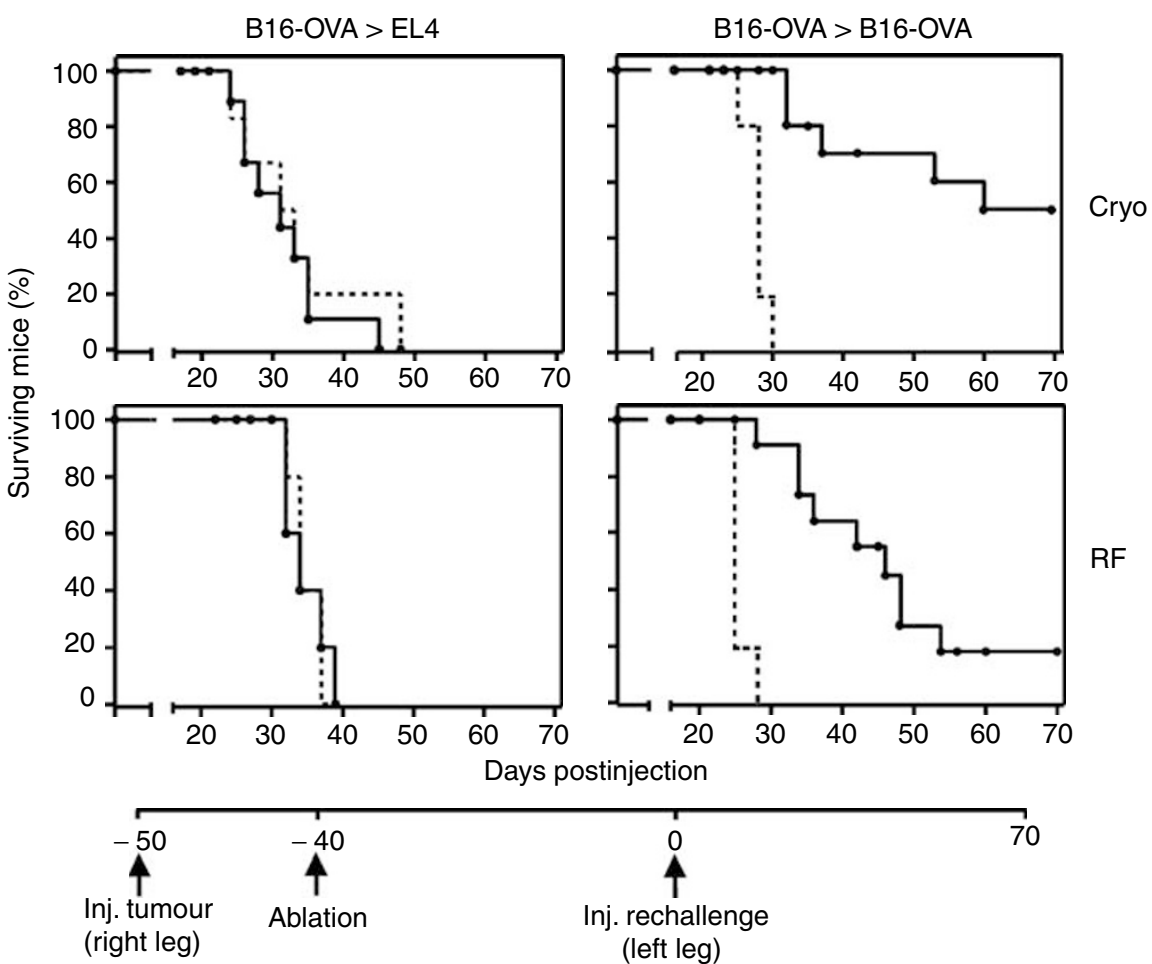

Figure I Anti-tumour immunity following radiofrequency or cryo ablation. Mice with established BI6-OVA melanomas (5-7 mm) were ablated by cryo (cryo, upper panels) or radiofrequency ablation (RF, lower panels). Forty days later, a re-challenge with $15 \times 10^{3} \mathrm{EL} 4$ (left panels) or I5 $\times 10^{3} \mathrm{BI}$ - 6 -OVA cells (right panels) was given s.c. in the contra-lateral leg. Figures depict survival curves demonstrating specificity of the response and growth reduction/protection after ablation. As a control, tumour growth was monitored by injection of the same tumour dose into naive mice (dotted lines). $T=0$ corresponds to the time of injection of the tumour re-challenge. $P<0.005$ for both BI 6 lines vs control. One out of four representative experiments is shown ( $n=5-I I$ ).

Applying magnetic bead sorting on the pan-DC marker CD11c we analysed the cells in the draining LN's containing ${ }^{111}$ In-KLH. As shown in Figure 2D, following ablation the cell-associated radioactivity was largely present in the $\operatorname{CD} 11 \mathrm{c}(+)$ fraction at 1 and even 3 days after ablation. Despite the fact that the CD11c + ( ) DC only comprised a minor fraction of total LN cells, they accounted for up to $25 \%$ of total cell-associated activity present in the LN's (cryo ablation, not shown). Consistent with Figure 2B, uptake of ${ }^{111} \mathrm{In}$ $\mathrm{KLH}$ following radiofrequency ablation was significantly lower compared to cryo ablation. The counts in the CD11c(-) fractions were all below 100 (not shown).

These data obtained using marker model antigens thus strongly suggest that also tumour-debris created by ablation acts as an antigen depot and that released antigens preferentially accumulate in CD11c $(+)$ DC in the draining LN. They also show that the method of destructing a tumour is highly relevant for the subsequent uptake dynamics of antigens.

\section{Accumulation of antigen-positive lymph node dendritic cell following ablation or conventional dendritic cell vaccination}

To compare the in vivo loading of DC by ablation with an externally loaded conventional DC vaccine, we analysed the numbers of antigen-positive LN DC after performing both techniques. In order to trace the antigen-experienced DC by flowcytometry, ovalbumin conjugated to the fluorophore Alexa488 (OVA-Alexa) was injected i.t. before ablation and DC derived from GFP-transgenic mice were used as an ex vivo DC vaccine. These GFP DC were matured with LPS, loaded with the relevant OVA-K ${ }^{\mathrm{b}}$ peptide (SIINFEKL) and $1 \times 10^{6} \mathrm{DC}$ were next injected s.c. or around an established $(5-7 \mathrm{~mm})$ B16-OVA tumour (p.t.). As shown in Figure $3 \mathrm{~A}$, over $12 \%$ of all draining LN CD11c(+) DC became OVA-Alexa $(+)$ after cryo ablation, whereas after radiofrequency ablation $7 \%$ of DC acquired the antigen. These data are in line with the accumulation of antigens in DC observed with

${ }^{111}$ In-proteins (see also Figure 2). Consistent with other studies (Josien et al, 2000; De Vries et al, 2003; Eggert et al, 2003), only small numbers $(<1 \%)$ of the ex vivo generated GFP DC reached the draining LN (Figure $3 \mathrm{~B}$ ). Moreover, plotting the absolute numbers of DC present in the draining LN further revealed a significant increase in total DC numbers after ablation, accompanied by an increased LN volume (Figure 3C and not shown). This implies that the absolute number of DC actually loaded with tumour antigen is even higher than can be concluded from the relative percentages of antigen-loaded DC shown in Figure 3A.

These data thus indicate that ablation is far more effective in obtaining marker antigen-loaded DC in the draining LN as compared to conventional DC vaccination.

\section{Ablation-dependent maturation of antigen-positive lymph node dendritic cell}

Next, we studied the maturation of DC in relation to antigen uptake. Therefore, OVA-Alexa $(+)$ or OVA-Alexa(-) (in vaccination: $\operatorname{GFP}(+)$ or $(-))$ DC were analysed for expression of the DC maturation marker CD80. OVA-Alexa $(+)$ DC showed a three-fold increase in CD80 expression relative to OVA-Alexa(-) DC in tumour-bearing and naïve mice (MFI's 987, 318 and 310, respectively) (Figure 4A). Moreover, CD80 expression further increased following cryo or radiofrequency ablation on OVAAlexa(+) DC but had no effect on OVA-Alexa(-) DC (MFI 1690 vs 362 and 1567 vs 361, respectively). This indicates that DC that acquired OVA-Alexa, as an indicator of tumour-debris, preferentially upregulate $\operatorname{CD} 80$, whereas this effect is further enhanced by ablation. Importantly, the induction of maturation by radio- 

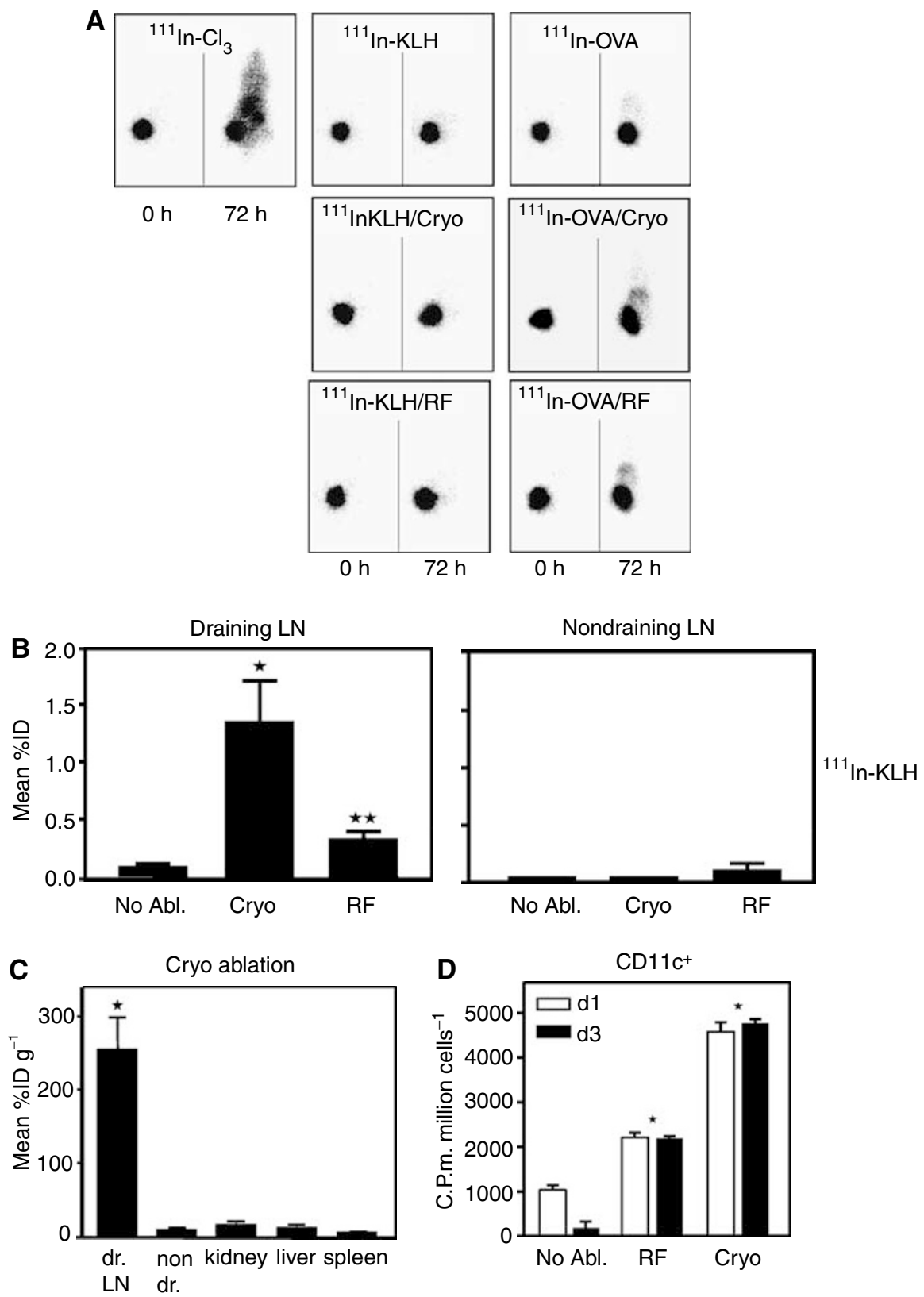

LN dr.

LN

Figure 2 Preferential uptake of tumour-derived antigens by LN DCs. (A) To study the fate of tumour antigens after ablation, $20 \mu \mathrm{Ci}$ of ${ }^{\prime \prime \prime}$ In-Cl (left panel), "II In-KLH (middle panels) or "I' In-OVA (right panels) was injected into established BI 6-OVA tumours (5-7 mm). Tumours were left untreated or ablated by cryo ablation (cryo) or radiofrequency ablation (RF) directly after these injections. $\gamma$-Camera imaging was performed at the indicated time points. For ${ }^{\prime \prime \prime}$ In-Cl ${ }_{3}$-injected mice the contours, tumour and liver are visible. One representative mouse out of three is shown. (B and $\left.\mathbf{C}\right)$ Biodistribution of ${ }^{\prime \prime \prime}$ In$\mathrm{KLH}$ was determined in dissected LNs and organs of mice injected i.t. I day before. Tumours were either left untreated or ablated directly after KLH injection. Radioactivity values from LN's of four mice per group are presented as mean percentages of injected dose with s.d., whereas the values for the organs are also corrected for weight. Mice in panel $\mathbf{C}$ received cryo ablation, but comparable results were obtained with RF ablation. (D) Lymph node suspensions from non-ablated and ablated mice (five mice pooled per group) that received i.t. ${ }^{111}$ In-KLH were subjected to magnetic bead sorting of $\mathrm{CDIIC}(+)$ cells. After sorting at days I and 3 after ablation, the cell-associated radioactivity was measured in the CDIIc(+) and CDIIc(-) (not shown) cell fraction. Values are presented as counts per minute, corrected for $1 \times 10^{6}$ cells and natural decay, with s.d. from triplicates. * $=P<0.005$ compared to no ablation. In all figures one out of three representative experiments is shown.

frequency ablation is equal to the induction by cryo ablation, even though less antigen uptake was observed. Analysis of the GFP-DC in the LN for CD80 expression, demonstrated that the exogenously loaded GFP $(+)$ DC were significantly more mature than the resident endogenous GFP(-) DC (Figure 4B) The observed MFI's were comparable to those seen on antigen-positive DC after ablation and were similar after p.t. or s.c. injection (Figure $4 \mathrm{~B}$ ).
Similar, but somewhat less profound results were obtained with CD86 expression (not shown).

The combined data thus not only indicate that ablation results in efficient DC loading but also that ablation-induced CD80 expression of the OVA-Alexa $(+)$ DC in the LN equals the $\mathrm{CD} 80$ expression on the DC from the LPS-matured ex vivo DC vaccine. 
A Nalive No ablation
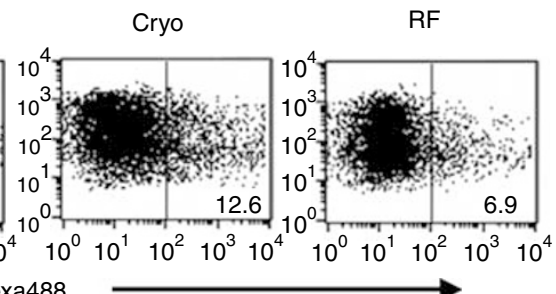

OVA-Alexa488

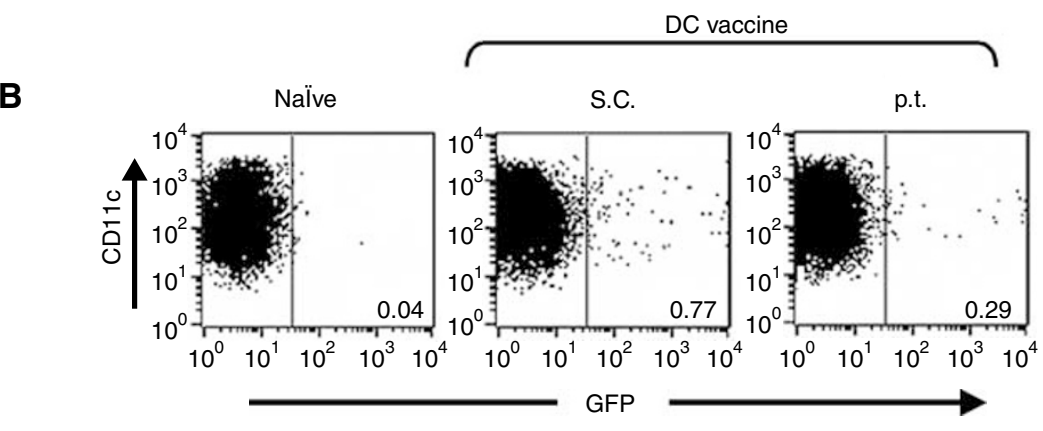

C

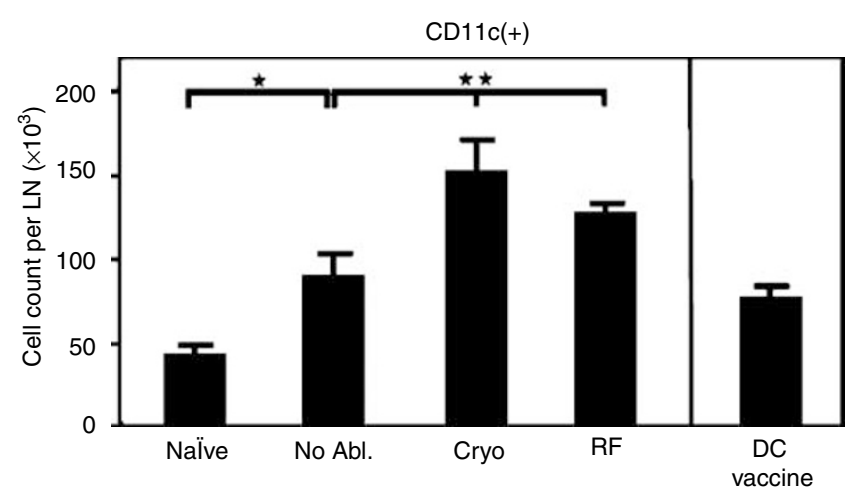

Figure 3 Increased numbers of antigen-positive DC in draining LN following ablation. (A) Fluorescence-activated cell sorting analysis of CDI Ic ++ ) DC isolated from pooled LN suspensions of naive, tumour-bearing or tumour-ablated mice ( $n=6$ per group). Mice received $20 \mu \mathrm{g}$ ovalbumin conjugated to Alexa-488 (OVA-Alexa488) i.t. just before the time point of ablation. Two days after the indicated treatments, CDII $(+)$ DC were isolated from draining LNs, stained for CDI Ic (clone HL3), gated and plotted for OVA-Alexa488 content. Values shown are percentages of OVA-Alexa488 + ) cells within the CDIIC (+) fraction. (B) Bone marrow dendritic cells were cultured from GFP-transgenic mice, loaded with peptides and matured ex vivo with LPS. Dendritic cells $\left(I \times 10^{6}\right)$ were injected p.t. in tumour-bearing mice or s.c. into naïve mice. Two days later CDIIC $(+)$ DC were isolated from pooled LN suspensions ( $n=6$ per group), stained, gated and plotted for GFP. (C) Absolute CDIIc $(+)$ cell count per LN. Data are obtained from experiments described in $A$ and $B$ and presented as means with s.d. from three independent experiments. As controls for all experiments, naïve mice were used that did not receive any injection (naïve). $*=P<0.005$ vs naïve, $* *=P<0.0$ l both vs no ablation.

\section{CTLA-4 blockade following ablation enhances systemic anti-tumour immunity}

We previously showed that immune modulation by blockade of CTLA-4 signalling enhances anti-tumour immunity after radiofrequency ablation. We now studied whether CTLA-4 blockade could be also used in the cryo ablation model. Figure 5 shows that the weak anti-tumour response observed after cryo ablation alone indeed could be enhanced by combination with CTLA-4 blockade. Comparable results were obtained when radiofrequency ablation was applied (Figure 5A). Control experiments showed that CTLA-4 injection alone had no significant effect on primary tumours or rechallenges (Figure 5B, left and right panel, respectively). Analysis of the mice for OVA-specific T cells revealed that far more specific $\mathrm{T}$ cells were present 10 days after the combination treatment as compared to ablation alone (Figure 5C). To determine the activation status of these T cells, IFN- $\gamma$ production was measured after activation with B16-OVA cells. Interestingly, $\mathrm{T}$ cells derived from mice treated with anti-CTLA-4 and ablation showed increased IFN- $\gamma$ production upon recognition of antigen compared to the IgG treatment (Figure 5D). In correspondence with the rechallenge model, somewhat less IFN- $\gamma$ producing, specific $\mathrm{T}$ cells were detected after radiofrequency ablation than after cryo ablation.

\section{Depletion of regulatory $\mathrm{T}$ cells before ablation enhances systemic anti-tumour immunity}

Next, we investigated whether depletion of regulatory $\mathrm{T}$ cells before ablation was also able to enhance tumour immunity. Hereto, mice received anti-CD25 antibodies 4 days before ablation of their B16-OVA tumours. The anti-CD25 antibodies did not have any effect on the outgrowth of the B16-OVA tumours, yielding similar tumour-sizes at the day of ablation (see also Figure 6B). Figure 6A shows that Treg depletion enhances the initially weak anti-tumour responses after both ablative techniques. This effect is comparable to the effects seen with anti-CTLA-4 treatment (see also Figure 5). Treg depletion alone had no significant effect on 


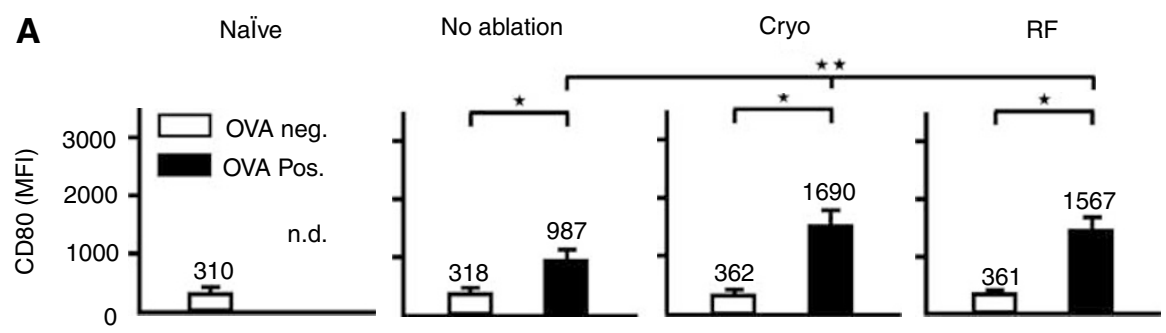

B

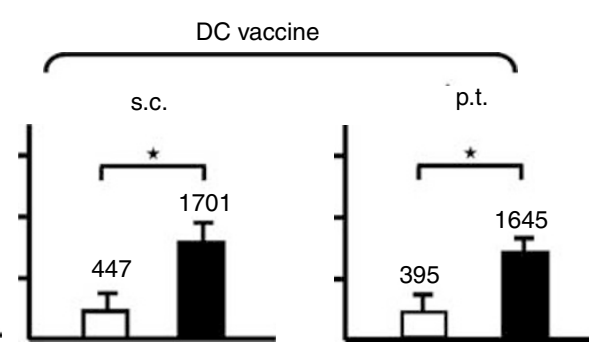

Figure 4 Ablation induces maturation of antigen-positive DC. OVA-Alexa488-positive or -negative CDI Ic $(+)$ cells $(\mathbf{A})$, or GFP-positive or negative CDIIC $(+)$ cells $(\mathbf{B})$, obtained in the experiments shown in Figure 3, were analysed separately for expression of the maturation marker CD80. Values indicated are mean MFl's with s.d. from three independent experiments. $*=P<0.005$, $* * *=0.05$ both vs no ablation.

primary tumours or re-challenges (Figure 6B). Tetramer analysis confirmed the presence of IFN- $\gamma$ producing, tumour-specific $\mathrm{T}$ cells after the combination treatment, whereas after each individual treatment these cells were absent (Figure 6C and D). In correspondence with the re-challenge model, somewhat less specific $\mathrm{T}$ cells were detected after radiofrequency ablation then after cryo ablation.

Collectively, these data thus suggest that antigen uptake following ablation does only lead to enhanced numbers of activated tumour-specific $\mathrm{T}$ cells, when suppressive regulation is switched off. In situ tumour destruction plus immune activation leads to a more potent systemic anti-tumour response than either treatment modality alone. This treatment regimen allows for direct antigen loading of DC in vivo without delivery of defined tumour antigens as in conventional DC vaccination.

\section{DISCUSSION}

Ex vivo generated mature DC have been shown to evoke tumourspecific responses in cancer patients (Figdor et al, 2004; Rosenberg et al, 2004). Dendritic cells vaccination is, however, time consuming and expensive, and in many cases the anti-tumour response falls short in strength to cure patients with established tumours. Herein, we report that tumour debris created by radiofrequency and cryo ablation comprises an effective antigen source for DC. Moreover, we show that tumour ablation could be efficiently combined with immune modulating approaches. This creates an effective 'in situ DC-vaccine' capable of inducing protection against lethal tumour re-challenges.

In situ tumour destruction with cryo, radiofrequency or laser ablation has received increasing attention as a treatment modality for focal cancer (Ruers et al, 2001; Ruers and Bleichrodt, 2002; Curley, 2003; Erce and Parks, 2003; Veth et al, 2005). However, little is known regarding the induction of immune responses after in situ tumour destruction or the fate of the generated tumour debris. In our experiments we applied a mouse B16 tumour model, in which ex vivo generated DC vaccines are mostly only effective in a prophylactic setting. Applying two types of ablation techniques and different types and labelled model antigens, we now demonstrated that antigen remaining in situ after tumour destruction creates an effective antigen depot for the induction of therapeutic anti-tumour immunity by DC. We applied two distinct exogenous antigens (OVA and $\mathrm{KLH}$ ) and two different approaches (radioactive and fluorescent labelling) to monitor the fate of antigens. Although it remains difficult to exclude that these model antigens do not behave like tumour debris in all facets, based on our as well as published results it is reasonable to assume that they provide relevant information regarding the flow of tumour debris (Itano et al, 2003). We showed that the majority of antigens remained at the ablated site and that very little antigen spreading was observed except to the draining LN. Within the draining LN, a large percentage of DC acquired antigen as soon as 1 day and for at least 3 days following ablation. The in vivo loading of DC upon cryo ablation was significantly more efficient than with radiofrequency ablation (Figures 2 and 3 ). The exact nature of this difference remains to be elucidated but is likely related to the kind of antigens that are created by the ablation and/ or to the endogenous signals that are produced upon tumour destruction.

Our results do not provide answers on how exactly DC acquire their antigens. It is, for instance, not known whether DC travel to the tumour and take up the antigens locally, or that the antigen floats to the LN via lymphatics, where LN resident DC engulf this material. According to a recent study demonstrating LN-DC that accumulated antigen deposited in s.c. tissue (Itano et al, 2003), both options might be occurring at the same time. The authors showed that antigen was first detected in LN-residing DC, followed by a second wave of antigen-positive DC that migrated from the periphery into the LN. Both waves were required for efficient immune response induction and were dependent on the presence of the challenge site. Our finding that both at day 1 and day 3 after ablation antigen-loaded DC could be discerned from the LN suggests that similar dynamics take place in our model. In this context, it is interesting to note that antigen loading in tumour bearing control mice seemed to decline in these 3 days (see also Figure 2D). Furthermore, we observed that antigens from the tumour depot preferentially accumulate in DC, but not in Bcells or macrophages (not shown). The basis for the observed antigen accumulation in DC remains to be studied in more detail, but is likely related to their strategic location within the LN and their ability to retain antigens within their endocytotic compartment, whereas macrophages rapidly degrade antigens in their lysosomes. 


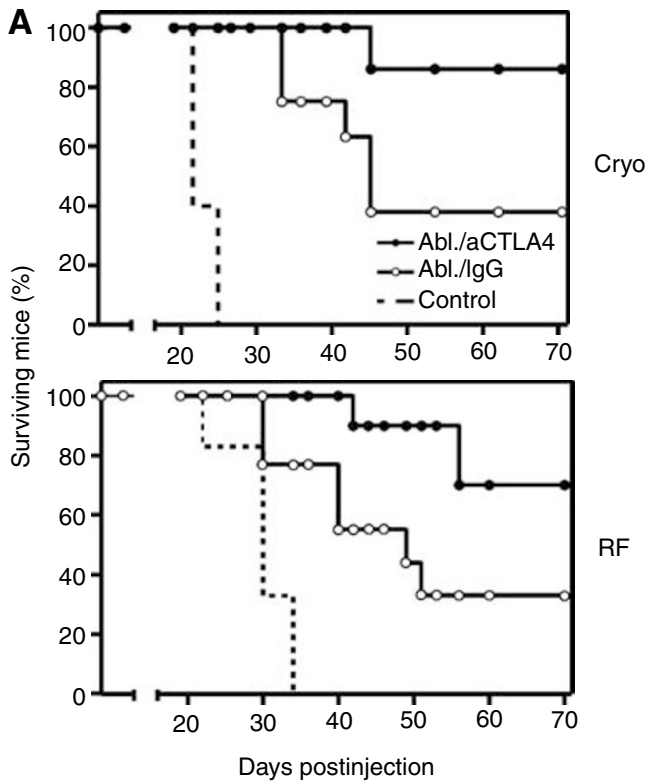

C

$\lg G$

Anti-CTLA-4
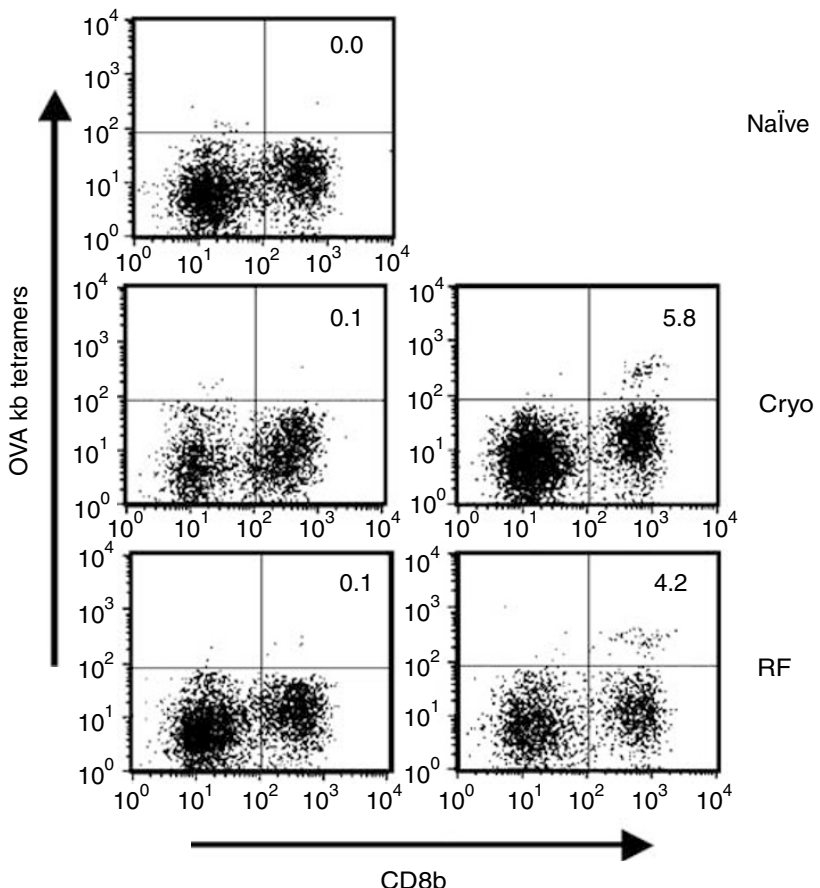

RF

$\mathrm{CD} 8 \mathrm{~b}$
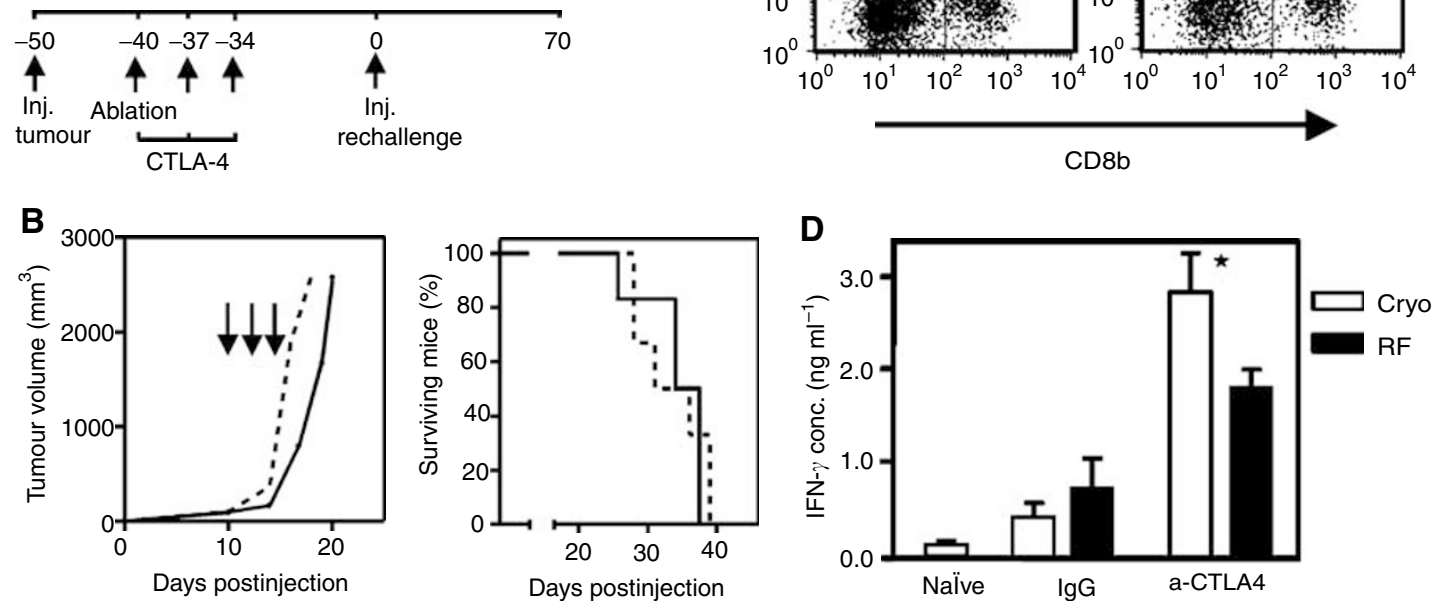

Figure 5 Anti-CTLA-4 improves therapeutic outcome of ablation. 0, 3 and 6 days after ablation of B I 6-OVA tumours, mice were injected i.p. with $200 \mu \mathrm{g}$ anti-CTLA-4 antibodies or control lgG. (A) Forty days following ablation of tumour-bearing mice receiving antibodies, a tumour re-challenge was performed as described before. Figures depict survival curves demonstrating growth reduction/protection after ablation plus CTLA-4 treatment. As a control, tumour growth was monitored by injection of the same tumour dose into naïve mice (dotted lines). $T=0$ corresponds to the time of injection of the tumour rechallenge. $P<0.05$ for CTLA-4 vs IgG in both cryo and RF figures. One out of three representative experiments is shown $(n=5-1 \mathrm{I})$. (B) Control experiment showing that CTLA-4 treatment by itself is insufficient to eradicate the primary tumour or re-challenges. Mice with established BI6OVA tumours (5-7 mm) were injected with $200 \mu \mathrm{g}$ anti-CTLA-4 antibodies at days 10, 13 or 16 after tumour inoculation (solid line, arrows) or PBS (dotted line). Next, tumour growth was monitored in time (left panel). CTLA-4 treatment or PBS given 40 days before a BI6OVA challenge (I5 $\times 10^{3}$ cells) did not affect survival of the mice (right panel). (C) At day 10 after ablation, a mix of $L N$ and spleen cells was obtained from mice treated as indicated. $T$ cells were harvested from spleen and LN and restimulated with irradiated, IFN- $\gamma$-treated BI 6-OVA cells and IL-2 for IO days, followed by staining with OVA tetramers $\left(K^{b}\right)$ and anti-CD8b. Depicted numbers represent the percentages of tetramer-positive cells within the CD8b + population. (D) T cells from the same bulk cultures were used for restimulation with BI6OVA cells. Supernatant from these cultures was harvested $24 \mathrm{~h}$ later and analysed for IFN- $\gamma$ content by standard ELISA methods. Shown are means with s.d. from triplicates, $*=P<0.05$ vs $\lg$. Experiments shown in figures (B -D) were repeated twice with comparable results.

The primary goal of conventional DC-based vaccination is to obtain tumour antigen-loaded DC in the draining LNs that are properly activated so that they do initiate immune responses. Although the nature of the antigens brought to the LN by DC loaded via ablation $v s$ antigen-positive DC from a conventional DC vaccine is difficult to compare, we could demonstrate that far more antigen-loaded DC were present in the draining LNs when radiofrequency or cryo ablation was performed (Figure 3). Moreover, both ablation procedures were able to significantly increase the absolute number of DC per LN, whereas vaccination was less able to do so (Figure 3C). Analysis of the maturation state of antigen-loaded and -unloaded DC in naïve, tumour-bearing and tumour-ablated mice revealed two interesting phenomena. First, DC that contained antigen expressed significantly higher levels of co-stimulatory molecules than antigen negative DC. These data are in line with in vitro data indicating that antigen uptake can affect DC activation (Randolph et al, 1998). Second, ablation resulted in a significant further increase in co-stimulatory molecule expression on antigen positive, but not antigen-negative DC. We note that neither antibodies present after positive MACS sorting, nor TLR ligands often present in OVA batches (Pasare and Medzhitov, 2004) did bias the CD80 staining on DC, as DC purified by negative selection showed similar results and OVA-Alexa did not mature DC in vitro (not shown). The exact nature of these ablation- 


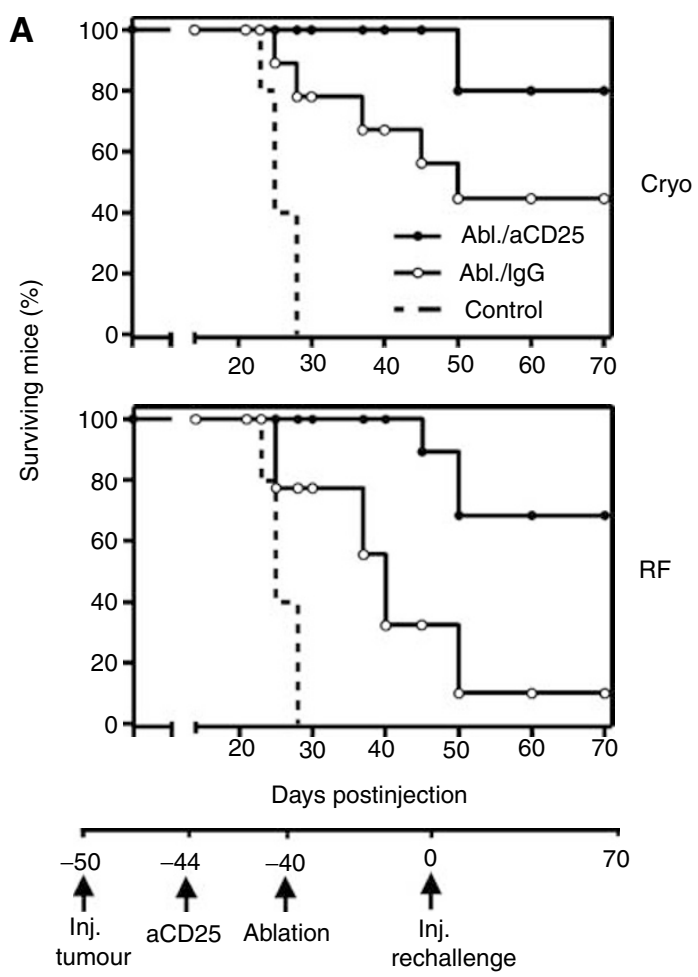

B

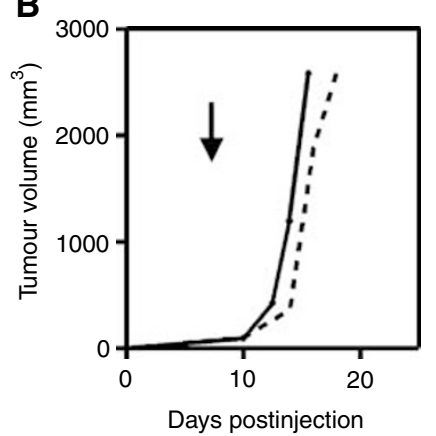

C $\lg G$

Anti-CD25

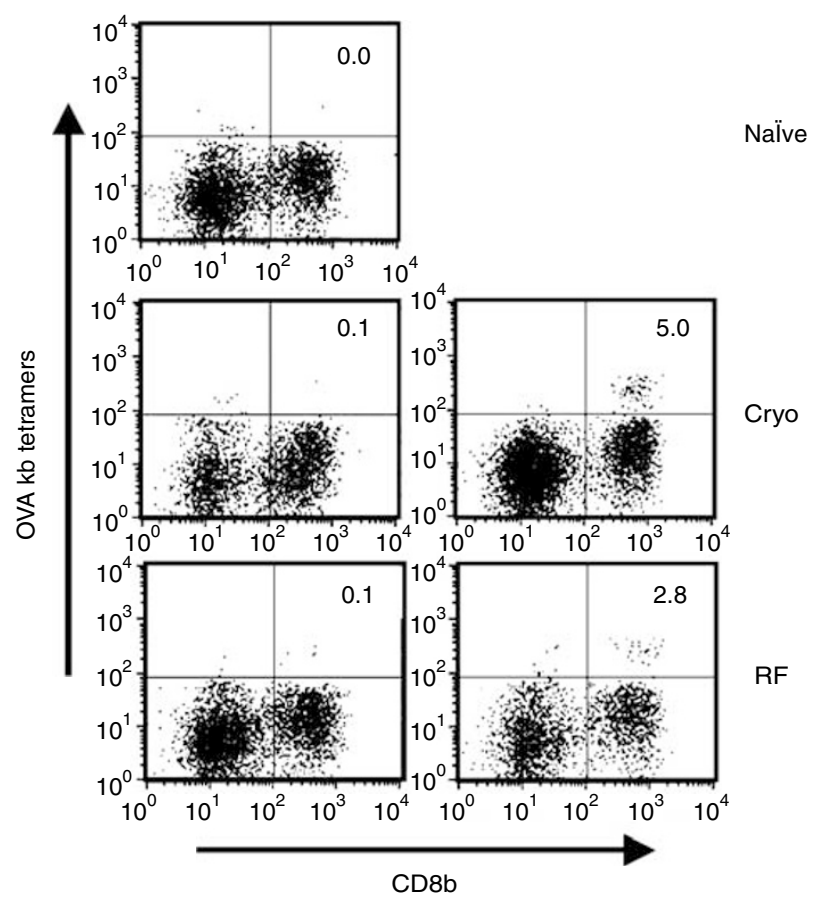

D
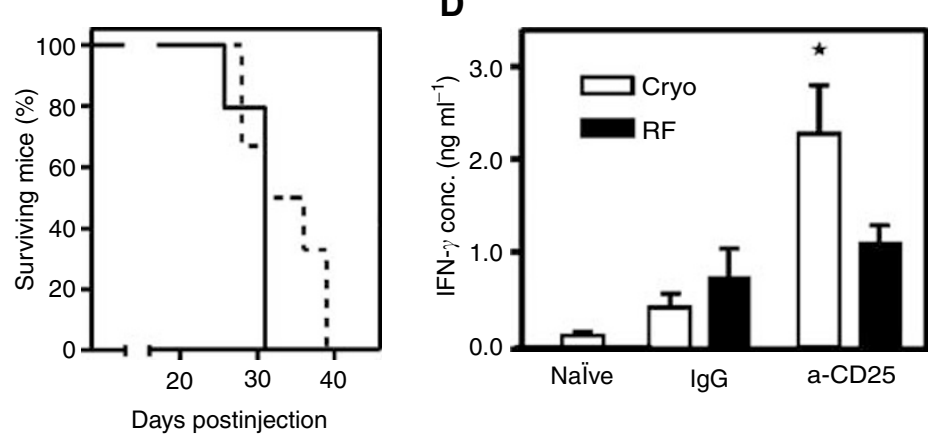

Figure 6 Depletion of regulatory $T$ cells improves therapeutic outcome of ablation. Four days before ablation of B I6-OVA tumours, mice were injected i.p. with $200 \mu \mathrm{g}$ anti-CD25 antibodies or control IgG. (A) Forty days following ablation of tumour-bearing mice receiving antibodies, a tumour re-challenge was performed as described before. Figures depict survival curves demonstrating growth reduction/protection after ablation plus Treg depletion. As a control, tumour growth was monitored by injection of the same tumour dose into naive mice (dotted lines). $T=0$ corresponds to the time of injection of the tumour re-challenge. $P<0.05$ for aCD25 vs IgG in both cryo and RF figures. One out of three representative experiments is shown ( $n=5-9$ ). (B) Control experiment showing that Treg depletion by itself is insufficient to eradicate the primary tumour or re-challenges. Mice with established BI6OVA tumours (5-7 mm) were injected with $200 \mu \mathrm{g}$ anti-CD25 antibodies (solid line, arrow) or PBS (dotted line). Next, tumour growth was monitored in time (left panel). Treg depletion or PBS given 40 days before a BI6OVA challenge $\left(15 \times 10^{3}\right.$ cells) did not affect survival of the mice (right panel). (C) At day I0 after ablation, a mix of LN and spleen cells was obtained from mice treated as indicated. $T$ cells were harvested from spleen and $L N$ and restimulated with irradiated, IFN- $\gamma$-treated B I6-OVA cells and IL-2 for 10 days, followed by staining with OVA tetramers (Kb) and anti-CD8b. Depicted numbers represent the percentages of tetramer-positive cells within the CD8b + population. (D) T cells from the same bulk cultures were used for restimulation with B $60 \mathrm{VA}$ cells. Supernatant from these cultures was harvested $24 \mathrm{~h}$ later and analysed for IFN- $\gamma$ content by standard ELISA methods. Shown are means with s.d. from triplicates, $*=P<0.05$ vs IgG. Experiments shown in figures $(\mathbf{B}-\mathbf{D})$, were repeated twice with comparable results.

dependent signals need further clarification, but may well represent cytokines or other endogenous mediators released after ablation (de Jong et al, 2001; Huang et al, 2002; Pasare and Medzhitov, 2004; Sporri and Reis e Sousa, 2005). It has, for instance, been shown that heat shock proteins and uric acid are present in cell debris, which both influence DC and other parts of the immune system (Somersan et al, 2001; Shi et al, 2003). Importantly, the observed ablation-induced increase in the number and maturation state of antigen-loaded DC is apparently not sufficient to induce complete tumour protection of mice.
Combination of ablation with in vivo immune modulation by either blockade of CTLA-4 signalling or depletion of regulatory T cells was shown to have beneficial effects in our re-challenge model. In both cases it provided a further delay in tumour growth compared to ablation alone. Importantly, only when CTLA-4 blockade or regulatory T-cell depletion were performed together with ablation, significant amounts of active OVA-specific T cells could be observed. It will be interesting to investigate the nature of the signals resulting in DC maturation and subsequent $\mathrm{T}$-cell expansion, and the possible effects of CTLA-4 blockade or Treg depletion on this. Moreover, possible future research can aim on 
combination of CTLA-4 blockade and Treg depletion. It was demonstrated in a murine tumour model that this combination had a striking synergistic effect on tumour immunity (Sutmuller et al, 2001).

Collectively, our data show that in vivo tumour destruction in combination with systemic immune modulation creates a unique and potent, 'in situ DC-vaccine'. Although radiofrequency ablation seems to be somewhat less efficient in loading DC compared to cryo ablation, both techniques can be efficiently combined with immune modulation. The fact that both ablative treatments as well as both the immune interventions are currently applied in cancer patients, makes this promising 'in vivo DC vaccine' readily applicable in clinical settings.

\section{ACKNOWLEDGEMENTS}

We thank the SPF department of the Nijmegen animal facility for technical assistance. This research is supported by grants from the Dutch Cancer Society (KWF 2003-2893, KWF-2005-3325).

\section{REFERENCES}

Adams S, O’Neill DW, Bhardwaj N (2005) Recent advances in dendritic cell biology. J Clin Immunol 25: 87-98

Banchereau J, Briere F, Caux C, Davoust J, Lebecque S, Liu YJ, Pulendran B, Palucka K (2000) Immunobiology of dendritic cells. Annu Rev Immunol 18: $767-811$

Banchereau J, Palucka AK (2005) Dendritic cells as therapeutic vaccines against cancer. Nat Rev Immunol 5: 296-306

Chen L, Ashe S, Brady WA, Hellstrom I, Hellstrom KE, Ledbetter JA, McGowan P, Linsley PS (1992) Costimulation of antitumor immunity by the B7 counterreceptor for the T lymphocyte molecules CD28 and CTLA4. Cell 71: $1093-1102$

Coulie PG, van der Bruggen P (2003) T-cell responses of vaccinated cancer patients. Curr Opin Immunol 15: $131-137$

Curley SA (2003) Radiofrequency ablation of malignant liver tumors. Ann Surg Oncol 10: $338-347$

de Jong KP, von Geusau BA, Rottier CA, Bijzet J, Limburg PC, de Vries EG, Fidler V, Slooff MJ (2001) Serum response of hepatocyte growth factor, insulin-like growth factor-I, interleukin-6, and acute phase proteins in patients with colorectal liver metastases treated with partial hepatectomy or cryosurgery. J Hepatol 34: 422-427

De Vries IJ, Krooshoop DJ, Scharenborg NM, Lesterhuis WJ, Diepstra JH, Van Muijen GN, Strijk SP, Ruers TJ, Boerman OC, Oyen WJ, Adema GJ, Punt CJ, Figdor CG (2003) Effective migration of antigen-pulsed dendritic cells to lymph nodes in melanoma patients is determined by their maturation state. Cancer Res 63: $12-17$

den Brok MH, Sutmuller RP, van der Voort R, Bennink EJ, Figdor CG, Ruers TJ, Adema GJ (2004) In situ tumor ablation creates an antigen source for the generation of antitumor immunity. Cancer Res 64: 4024-4029

Egen JG, Kuhns MS, Allison JP (2002) CTLA-4: new insights into its biological function and use in tumor immunotherapy. Nat Immunol 3: 611-618

Eggert AA, Schreurs MW, Boerman OC, Oyen WJ, de Boer AJ, Punt CJ, Figdor CG, Adema GJ (1999) Biodistribution and vaccine efficiency of murine dendritic cells are dependent on the route of administration. Cancer Res 59: $3340-3345$

Eggert AA, van der Voort R, Torensma R, Moulin V, Boerman OC, Oyen WJ, Punt CJ, Diepstra H, de Boer AJ, Figdor CG, Adema GJ (2003) Analysis of dendritic cell trafficking using EGFP-transgenic mice. Immunol Lett 89: $17-24$

Erce C, Parks RW (2003) Interstitial ablative techniques for hepatic tumours. Br J Surg 90: 272-289

Falo Jr LD, Kovacsovics-Bankowski M, Thompson K, Rock KL (1995) Targeting antigen into the phagocytic pathway in vivo induces protective tumour immunity. Nat Med 1: 649-653

Figdor CG, de Vries IJ, Lesterhuis WJ, Melief CJ (2004) Dendritic cell immunotherapy: mapping the way. Nat Med 10: $475-480$

Furumoto K, Soares L, Engleman EG, Merad M (2004) Induction of potent antitumor immunity by in situ targeting of intratumoral DCs. J Clin Invest 113: $774-783$

Garcea G, Lloyd TD, Aylott C, Maddern G, Berry DP (2003) The emergent role of focal liver ablation techniques in the treatment of primary and secondary liver tumours. Eur J Cancer 39: 2150-2164

Huang A, McCall JM, Weston MD, Mathur P, Quinn H, Henderson DC, Allen-Mersh TG (2002) Phase I study of percutaneous cryotherapy for colorectal liver metastasis. Br J Surg 89: $303-310$

Itano AA, McSorley SJ, Reinhardt RL, Ehst BD, Ingulli E, Rudensky AY, Jenkins MK (2003) Distinct dendritic cell populations sequentially present antigen to CD4 T cells and stimulate different aspects of cellmediated immunity. Immunity 19: 47-57
Josien R, Li HL, Ingulli E, Sarma S, Wong BR, Vologodskaia M, Steinman RM, Choi Y (2000) TRANCE, a tumor necrosis factor family member, enhances the longevity and adjuvant properties of dendritic cells in vivo. J Exp Med 191: 495-502

Onizuka S, Tawara I, Shimizu J, Sakaguchi S, Fujita T, Nakayama E (1999) Tumor rejection by in vivo administration of anti-CD25 (interleukin-2 receptor alpha) monoclonal antibody. Cancer Res 59: 3128-3133

Pasare C, Medzhitov R (2004) Toll-dependent control mechanisms of CD4 $\mathrm{T}$ cell activation. Immunity 21: $733-741$

Raj GV, Reddan DJ, Hoey MB, Polascik TJ (2003) Management of small renal tumors with radiofrequency ablation. Urology 61: 23-29

Randolph GJ, Beaulieu S, Lebecque S, Steinman RM, Muller WA (1998) Differentiation of monocytes into dendritic cells in a model of transendothelial trafficking. Science 282: 480-483

Rosenberg SA, Yang JC, Restifo NP (2004) Cancer immunotherapy: moving beyond current vaccines. Nat Med 10: 909-915

Ruers T, Bleichrodt RP (2002) Treatment of liver metastases, an update on the possibilities and results. Eur J Cancer 38: 1023-1033

Ruers TJ, Joosten J, Jager GJ, Wobbes T (2001) Long-term results of treating hepatic colorectal metastases with cryosurgery. Br J Surg 88: 844-849

Sakaguchi S (2005) Naturally arising Foxp3-expressing CD25+CD4+ regulatory $\mathrm{T}$ cells in immunological tolerance to self and non-self. Nat Immunol 6: $345-352$

Schuler G, Schuler-Thurner B, Steinman RM (2003) The use of dendritic cells in cancer immunotherapy. Curr Opin Immunol 15: 138-147

Shi Y, Evans JE, Rock KL (2003) Molecular identification of a danger signal that alerts the immune system to dying cells. Nature 425: 516-521

Somersan S, Larsson M, Fonteneau JF, Basu S, Srivastava P, Bhardwaj N (2001) Primary tumor tissue lysates are enriched in heat shock proteins and induce the maturation of human dendritic cells. J Immunol 167: $4844-4852$

Sporri R, Reis e Sousa C (2005) Inflammatory mediators are insufficient for full dendritic cell activation and promote expansion of CD4+ T cell populations lacking helper function. Nat Immunol 6: 163-170

Steinman RM, Mellman I (2004) Immunotherapy: bewitched, bothered, and bewildered no more. Science 305: 197-200

Steinman RM, Pope M (2002) Exploiting dendritic cells to improve vaccine efficacy. J Clin Invest 109: 1519-1526

Sutmuller RP, van Duivenvoorde LM, van Elsas A, Schumacher TN, Wildenberg ME, Allison JP, Toes RE, Offringa R, Melief CJ (2001) Synergism of cytotoxic T lymphocyte-associated antigen 4 blockade and depletion of $\mathrm{CD} 25(+)$ regulatory $\mathrm{T}$ cells in antitumor therapy reveals alternative pathways for suppression of autoreactive cytotoxic $\mathrm{T}$ lymphocyte responses. J Exp Med 194: 823-832

Townsend SE, Allison JP (1993) Tumor rejection after direct costimulation of CD8+ T cells by B7-transfected melanoma cells. Science 259: $368-370$

Trinchieri G (2003) Interleukin-12 and the regulation of innate resistance and adaptive immunity. Nat Rev Immunol 3: 133-146

Veth R, Schreuder B, van Beem H, Pruszczynski M, de Rooy J (2005) Cryosurgery in aggressive, benign, and low-grade malignant bone tumours. Lancet Oncol 6: 25-34

Vremec D, Pooley J, Hochrein H, Wu L, Shortman K (2000) CD4 and CD8 expression by dendritic cell subtypes in mouse thymus and spleen. $J$ Immunol 164: 2978-2986

Zagoria RJ, Chen MY, Kavanagh PV, Torti FM (2001) Radio frequency ablation of lung metastases from renal cell carcinoma. J Urol 166: $1827-1828$ 